\title{
Rassegna giurisprudenziale in tema di danno biologico: recentissime novità in tema di micro e macropermanenti e rapporti tra danno biologico e immissioni
}

\author{
Giovanni Cicchitelli \\ Avvocato, Criminologo, Roma, Italy
}

A partire da questo numero della presente Rivista, viene introdotta una nuova rubrica: la "rassegna giurisprudenziale". Essa riguarderà, di volta in volta, temi in linea con l'oggetto della Rivista stessa, di verosimile interesse per il lettore.

Lo scorso mese di agosto è stato pregno di importanti interventi normativi -conseguenza a loro volta delle evoluzioni giurisprudenziali, specie della Cassazione -in tema di danno biologico, quest'ultimo da intendersi quale lesione temporanea o permanente all'integrità psico-fisica della persona suscettibile di accertamento medico-legale che esplica un'incidenza negativa sulle attività quotidiane e sugli aspetti dinamico-relazionali della vita del danneggiato, indipendentemente da eventuali ripercussioni sulla sua capacità di produrre reddito (è noto l'indubbio apporto fornito a tale definizione dagli artt. 138 e 139 del Codice delle assicurazioni private, cioè dal D.lgs. n. 209/2005, nonché dall'esegesi nelle ormai storiche sentenze delle Sezioni Unite della S.C. di Cassazione nn. 26972-26975/2008).

Anzitutto, con l'art. 1, comma 17, della Legge 04/08/2017, $\mathrm{n}^{\circ}$ 124, G.U. 14/08/2017 (c.d. "legge sulla concorrenza", che tante polemiche ha suscitato per altri aspetti) è stato modificato l'art. 138 del Codice delle assicurazioni private (D.lgs. n. 209/2005): la stima delle $\mathrm{cd}$. macrolesioni dovrà avvenire attraverso una tabella unica per la valutazione delle lesioni aventi tra i 10 e i 100 punti di invalidità permanente (eliminando ex lege il "contrasto" tra le tabelle dei Tribunali di Roma e Milano); detta tabella unica monetaria del risarcimento del danno biologico uguale per tutti (ma solo per i sinistri automobilistici, si badi) dovrà essere predisposta ed approvata entro 120 giorni (indicativi e non tassativi) dalla pubblicazione della legge, con decreto del Presidente della Repubblica, tenendo conto dei "criteri di valutazione del danno non patrimoniale ritenuti congrui dalla consolidata giurisprudenza di legittimità", cioè della Cassazione.

In pratica, le Tabelle del Tribunale di Milano per il risarcimento del danno alla persona delle vittime della strada in ambito di sinistri automobilistici diventano le tabelle legislative uniche nazionali, come già indicato dalla Cassazione stessa (cfr., ex plurimis, Cassazione civile, sez. III, 20/04/2017, n. 9950, in Diritto \& Giustizia 2017, 21 aprile (nota di: Renato Savoia, il quale sottolinea "la riaffermazione del principio tabellare milanese come metodo ritenuto in grado di soddisfare nel miglior modo possibile la parità di trattamento"); si vedano anche Cass. 12408/2011 e Cass. 10263/2015).

Peraltro, ai sensi del comma 18 della Legge 04/08/2017 "la tabella unica nazionale predisposta con il decreto del Presidente della Repubblica di cui all'articolo 138, comma 1, del codice delle assicurazioni private, di cui al decreto legislativo 7 settembre 2005, n. 209, come sostituito dal comma 17 del presente articolo, si applica ai sinistri e agli eventi verificatisi successivamente alla data di entrata in vigore del medesimo decreto del Presidente della Repubblica".
Come sottolinea il Cesari nella rivista online Altalex "è $d a$ auspicare che la medicina legale italiana e la psicologia tramite le società scientifiche di riferimento ottengano la necessaria attenzione di riferimento per una tabella delle menomazioni che rispetti la evoluzione scientifica dell'ultimo decennio" (cfr. http://www.altalex.com/documents/news/2017/08/03/danno-biologico-via-liberaalla-tabella-unica-nazionale-ma-solo-per-i-sinistri).

Per quanto riguarda, invece, l'art. 139 del Codice delle assicurazioni private (Danno non patrimoniale per lesioni di lieve entità, cd. micropermanenti), esso è stato sostituito dall'art. 1, comma 19, della Legge 04/08/2017, $n^{\circ} 1241$.

Infine, resta da segnalare la sempre agostana Cassazione Civile, sez. II, ordinanza 28/08/2017 n² 20445, che torna sul rapporto tra immissioni e danno biologico.

Ebbene, 1'art. $844^{2}$ del codice civile (topograficamente collocato nel titolo, II dedicato alla proprietà), statuisce che "il proprietario di un fondo non può impedire le immissioni di fumo o calore, le esalazioni, i rumori, gli scuotimenti e simili propagazioni derivanti dal fondo del vicino, se non superano la normale tollerabilità, avuto riguardo anche alla condizione dei luoghi". Soggiunge l'articolo che "Nell'applicare questa norma l'autorità giudiziaria deve contemperare le esigenze della produzione con le ragioni della proprietà. Può tener conto della priorità di un determinato uso".

1 1. Il risarcimento del danno biologico per lesioni di lieve entità, derivanti da sinistri conseguenti alla circolazione di veicoli a motore e di natanti, è effettuato secondo i criteri e le misure seguenti: a) a titolo di danno biologico permanente, è liquidato per i postumi da lesioni pari o inferiori al 9 per cento un importo crescente in misura più che proporzionale in relazione a ogni punto percentuale di invalidità; tale importo è calcolato in base all'applicazione a ciascun punto percentuale di invalidità del relativo coefficiente secondo la correlazione stabilita dal comma 6 . L'importo così determinato si riduce con il crescere dell'età del soggetto in ragione dello 0,5 per cento per ogni anno di età a partire dall'undicesimo anno di età. Il valore del primo punto è pari a 795,91 euro; b) a titolo di danno biologico temporaneo, è liquidato un importo di 39,37 euro per ogni giorno di inabilità assoluta; in caso di inabilità temporanea inferiore al 100 per cento, la liquidazione avviene in misura corrispondente alla percentuale di inabilità riconosciuta per ciascun giorno. 2. Ai fini di cui al comma 1, per danno biologico si intende la lesione temporanea o permanente all'integrità psico-fisica della persona, suscettibile di accertamento medico-legale, che esplica un'incidenza negativa sulle attività quotidiane e sugli aspetti dinamico-relazionali della vita del danneggiato, indipendentemente da eventuali ripercussioni sulla sua capacità di produrre reddito. In ogni caso, le lesioni di lieve entità, che non siano suscettibili di accertamento clinico strumentale obiettivo, ovvero visivo, con riferimento alle lesioni, quali le cicatrici, oggettivamente riscontrabili senza 1'ausilio di strumentazioni, non possono dar luogo a risarcimento per danno biologico permanente. 
Senza addentrarci in complesse questioni esegetiche dell'art. 844 c.c., che peraltro esulerebbero dall'oggetto della disamina, e cioè il rapporto che la Cassazione ha individuato (recte: ribadito) tra immissioni e danno biologico, basti qui rammentare che " $L a$ disciplina relativa alle immissioni moleste provenienti dal fondo vicino, dettata dall'art. 844 c.c., ed il limite della tutela inibitoria alle immissioni che superano la normale tollerabilità, trovano applicazione anche nei rapporti di condominio" (così, ex multiis, Cassazione civile, sez. II, 06/04/1983, n. 2396).

La Corte d'Appello di Roma, in accoglimento dell'appello proposto, aveva riformato la sentenza di prime cure, negando ad una condomina il diritto al risarcimento del danno da immissioni rumorose provenienti da una falegnameria, poiché risarcibile, a parere della corte di merito, "solo ove ne sia derivata comprovata lesione alla salute, non essendo risarcibile la minore godibilità della vita", da provare viepiù con "idonea documentazione sanitaria e chiedere l'espletamento di una c.t.u. medico-legale".

La S.C. di Cassazione, invece, ha accolto il ricorso proposto dalla condomina, cassando l'impugnata sentenza e, pronunciando nel merito, accoglieva la domanda attrice, condannando ad un risarcimento di $€ 10.000$ parte resistente.

Ed invero, secondo il giudice di legittimità "il danno non patrimoniale conseguente ad immissioni illecite è risarcibile indipendentemente dalla sussistenza di un danno biologico documentato, quando sia riferibile alla lesione del diritto al normale svolgimento della vita privata e familiare all'interno di un'abitazione e, comunque, del diritto alla piena e libera esplicazione delle proprie abitudini di vita".

La riaffermazione di detto principio (già contenuto, ad esempio, in Cassazione civile, sezione II, 19 agosto 2011, n. 17427, all'indomani il nuovo "statuto"di risarcimento del danno non patrimoniale inaugurato con le quattro decisioni delle Sezioni Unite del 2008 dianzi cennate), ha importanti riflessi probatori: la prova del pregiudizio subito può essere fornita anche mediante presunzioni o sulla base delle nozioni di comune esperienza; e, ciò, nella misura in cui si tratta di diritti costituzionalmente garantiti, la cui tutela è ulteriormente rafforzata dall'art. 8 della Convenzione europea dei diritti dell'uomo, norma alla quale il giudice interno è tenuto a conformarsi (vedi Cass. 16/10/2015, n. 20927); viceversa, per il danno non patrimoniale da lesione del diritto alla salute (c.d. danno biologico), configurabile quando le immissioni intollerabili cagionano a chi le subisce una vera e propria patologia (documentata da certificazioni

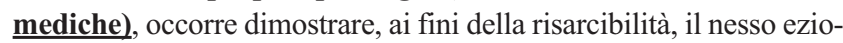
logico tra le immissioni e la malattia riscontrata (si veda sul punto Cassazione Civile, sez. II, ordinanza 28/08/2017, n $^{\circ} 20445$, con nota di Tiziana di Mauro, http:/www.altalex.com/documents/news/ 2017/08/31/immissioni-rumorose).
Correspondence: Giovanni Cicchitelli.

E-mail: giovannicicchitelli@gmail.com

Rubrica a cura di Gianluca Montanari Vergallo, Giovanni Cicchitelli e Giovanni Neri.

Received for publication: 5 October 2017.

Revision received: 6 November 2017.

Accepted for publication: 3 December 2017.

(C) Copyright G. Cicchitelli, 2017

Licensee PAGEPress, Italy

Rivista di Psicopatologia Forense, Medicina Legale, Criminologia

2017; $22: 44-45$

\begin{abstract}
3. Qualora la menomazione accertata incida in maniera rilevante su specifici aspetti dinamico-relazionali personali documentati e obiettivamente accertati ovvero causi o abbia causato una sofferenza psico-fisica di particolare intensità, l'ammontare del risarcimento del danno, calcolato secondo quanto previsto dalla tabella di cui al comma 4, può essere aumentato dal giudice, con equo e motivato apprezzamento delle condizioni soggettive del danneggiato, fino al 20 per cento. L'ammontare complessivo del risarcimento riconosciuto ai sensi del presente articolo è esaustivo del risarcimento del danno non patrimoniale conseguente a lesioni fisiche. 4. Con decreto del Presidente della Repubblica, previa deliberazione del Consiglio dei ministri, su proposta del Ministro della salute, di concerto con il Ministro del lavoro e delle politiche sociali, con il Ministro della giustizia e con il Ministro dello sviluppo economico, si provvede alla predisposizione di una specifica tabella delle menomazioni dell'integrità psico-fisica comprese tra 1 e 9 punti di invalidità. 5. Gli importi indicati nel comma 1 sono aggiornati annualmente con decreto del Ministro dello sviluppo economico, in misura corrispondente alla variazione dell'indice nazionale dei prezzi al consumo per le famiglie di operai e impiegati accertata dall'ISTAT. 6. Ai fini del calcolo dell'importo di cui al comma 1, lettera a), per un punto percentuale di invalidità pari a 1 si applica un coefficiente moltiplicatore pari a 1 , per un punto percentuale di invalidità pari a 2 si applica un coefficiente moltiplicatore pari a 1,1 , per un punto percentuale di invalidità pari a 3 si applica un coefficiente moltiplicatore pari a 1,2 , per un punto percentuale di invalidità pari a 4 si applica un coefficiente moltiplicatore pari a 1,3, per un punto percentuale di invalidità pari a 5 si applica un coefficiente moltiplicatore pari a 1,5 , per un punto percentuale di invalidità pari a 6 si applica un coefficiente moltiplicatore pari a 1,7 , per un punto percentuale di invalidità pari a 7 si applica un coefficiente moltiplicatore pari a 1,9 , per un punto percentuale di invalidità pari a 8 si applica un coefficiente moltiplicatore pari a 2,1 e per un punto percentuale di invalidità pari a 9 si applica un coefficiente moltiplicatore pari a 2,3.

2 Oltre all'art. 844 del codice civile che vieta le propagazioni di rumori che superano la normale tollerabilità, avuto però riguardo alla condizione dei luoghi e al "preuso" è utile tenere conto anche di quanto disposto dal decreto legislativo 27 gennaio 1992, n. 134 (rumore aereo emesso dagli apparecchi domestici); dalla Legge 26 ottobre 1995, n. 447 (legge quadro sull'inquinamento acustico); dal DPCM 16 aprile 1999, n. 215 (norme sui requisiti acustici delle sorgenti sonore nei pubblici esercizi); dal Decreto legislativo 19 agosto 2005, n. 194 (in attuazione della direttiva 2002/49/CE in materia di rumore ambientale).
\end{abstract}

